\title{
On the Modeling of Uplink Inter-Cell Interference based on Proportional Fair Scheduling
}

\author{
Hina Tabassum\#, Ferkan Yilmaz ${ }^{\#}$, Zaher Dawy*, and Mohamed Slim Alouini" \\ \#Electrical Engineering Program, King Abdullah University of Science and Technology (KAUST), \\ Thuwal, Mekkah Province, Saudi Arabia \\ * Department of Electrical and Computer Engineering, American University of Beirut (AUB), \\ Beirut, Lebanon \\ \{hina.tabassum, ferkan.yilmaz, slim.alouini\}@kaust.edu.sa, zaher.dawy@aub.edu.lb
}

\begin{abstract}
We derive a semi-analytical expression for the uplink inter-cell interference (ICI) assuming proportional fair scheduling (with a maximum normalized signal-to-noise ratio (SNR) criterion) deployed in the cellular network. The derived expression can be customized for different models of channel statistics that can capture path loss, shadowing, and fading. Firstly, we derive an expression for the distribution of the locations of the allocated user in a given cell. Then, we derive the distribution and moment generating function of the uplink ICI from one interfering cell. Finally, we determine the moment generating function of the cumulative uplink ICI from all interfering cells. The derived expression is utilized to evaluate important network performance metrics such as outage probability and fairness among users. The accuracy of the derived expressions is verified by comparing the obtained results to Monte Carlo simulations.
\end{abstract}

\section{INTRODUCTION}

Modeling the inter-cell interference (ICI) in wireless cellular networks plays an imperative role in evaluating the system performance metrics, devising efficient resource allocation schemes, and developing interference mitigation techniques. In OFDMA cellular networks, the system bandwidth is decomposed into flat-faded orthogonal subcarriers. These subcarriers are adaptively allocated among users within a cell based on a predefined scheduling scheme. Considering the orthogonality among the allocated users in a given cell, the intra-cell interference can be ignored. However with frequency reuse factor equal to one, the users allocated on the same subcarrier in neighboring cells can cause severe ICI, depending on their distance from the base station (BS) of interest. Since each subcarrier is allocated to only one user per cell and the number of interfering cells is relatively limited, it may not be accurate to model ICI as a Gaussian random variable (RV) by invoking the central limit theorem.

Numerous interference models are available in the literature for various intended applications, different environments and scenarios. Several recent studies considered the modeling of ICI in the downlink of cellular networks where the location of interferers is usually deterministic [1]-[4]. However, compared to the downlink, the modeling of ICI in the uplink is more challenging due to the arbitrary locations of the interferers. The number of significantly contributing interferers in the uplink can vary based on the user's locations which is highly dependent on the deployed scheduling scheme. In [5], the authors developed an analytical model for subcarrier collisions as a function of the cell load and frequency reuse scheme. They derived an expression for the SINR in the uplink and downlink, ignoring the effect of shadowing and fading. In [6], the authors developed an exact analytical expression for the subcarrier collision probability considering non-coordinated schedulers. In [7], the authors modeled uplink ICI in an OFDMA network as a function of the reuse partitioning radius and traffic load assuming arbitrary scheduling. In [8], the authors presented a semi-analytical method to approximate the distribution of uplink ICI through numerical simulations without considering the impact of scheduling schemes. In [9], we presented a semianalytical approach to derive the distribution of uplink ICI assuming greedy scheduling in all cells.

In this work, we generalize the approach presented in [9] to derive a semi-analytical expression for the uplink ICI on a given subcarrier assuming proportional fair scheduling with a maximum normalized SNR criterion. Proportional fair scheduling is widely used in practice due to its ability to enhance network throughput compared to round robin scheduling while taking into account fairness among users with different locations and channel conditions. The derived expressions are then utilized to evaluate important network performance metrics such as outage probability and average fairness.

The remainder of this paper is organized as follows. Section II describes the system model and presents the approach used to derive the uplink ICI expression. In Section III, we determine the distribution of the locations of the allocated user in a given cell. Based on this, in Section IV, we determine the distribution of the uplink ICI from a neighboring cell, compute the moment generating function (MGF) of the cumulative ICI from all interfering cells, and address different fading scenarios. In Section V, we compute important network performance metrics based on the derived MGF. Section VI presents selected numerical and simulation results followed by concluding remarks in Section VII.

Notation: Throughout the paper, $\operatorname{Exp}(\lambda)$ denotes an exponential distribution with parameter $\lambda$, $\operatorname{Gamma}\left(m_{s}, m_{c}\right)$ represents a Gamma distribution with shape parameter $m_{s}$ and scale parameter $m_{c} . \Gamma($.$) represents the Gamma function. p(A)$ denotes the probability of event A. $f($.$) and F($.$) denote the$ probability distribution function and cumulative distribution function, respectively. $[a, b]$ denotes a discrete set of elements which ranges from $a$ to $b$. Finally, $\mathbb{E}[$.$] denotes the expectation.$

\section{System Model ANd PROPOSED APPROACH}

We consider a given cell surrounded by $L$ interfering cells. For analytical convenience, the cells are assumed to be circular with radius $R$. Each cell $l$ is assumed to have $U$ users uniformly distributed. Without loss of generality, the average transmit power of each user in the uplink is assumed to be unity. We assume users transmit with maximum power with rate adaptation depending on their channel qualities. Thus, each user has an instantaneous SNR $\gamma$ based on its location, shadowing, and fading statistics:

$$
\gamma=r^{-\beta} \zeta
$$

where $r$ is the user distance from its serving BS, $\beta$ is the path loss exponent, and $\zeta$ is a random variable that represents combined shadowing and fading statistics.

Each cell is decomposed into $K$ concentric circular rings. The circular regions between two adjacent rings are characterized by uniform path loss variation (in $\mathrm{dB}$ ) and, thus, possess non-uniform width $\Delta_{k}$. Since path loss varies exponentially 
with distance, $\Delta_{k}$ increases from cell center to cell edge. Thus, the number of circular regions in each cell depends on the path loss exponent. The average number of users in a given ring $k$ can be computed as follows:

$$
u_{k}=\frac{U\left(r_{k}^{2}-r_{k-1}^{2}\right)}{R^{2}} \quad k=1,2, \cdots, K,
$$

where $r_{k}$ denotes the radius of ring $k$. It is important to note that $u_{k}$ can be a fraction of a number; therefore, we round off the fractional part of users in each ring. The motivation behind dividing each cell into a number of circular regions is that in each region the channel conditions of the users becomes relatively similar especially for large values of $K$.

The proposed approach to model ICI is detailed in the following steps:

1) Derive the distribution $f_{r_{\mathrm{sel}}}(r)$ of allocating the given subcarrier to a user at a distance $r_{\text {sel }}$ from its serving BS considering proportional fair scheduling with maximum normalized SNR criterion. For simplicity, the scheduling scheme is considered to be the same in all cells, therefore $f_{r_{\text {sel }}}(r)$ applies to all cells

2) Derive the distribution of the distance between the allocated users in the neighbor interfering cells and the BS of the cell of interest, i.e., determine $f_{\tilde{r}_{\text {sel }}}(\tilde{r})$ using $f_{r_{\text {sel }}}(r)$ where $\tilde{r}_{\text {sel }}$ is the distance between interfering users and the BS of interest.

3) Derive the distribution of the interference $f_{X_{l}}(x)$ from the allocated user in neighboring cell $l$ to the BS of interest. $X_{l}$ can be written as follows:

$$
X_{l}=\tilde{r}_{\text {sel }}^{-\beta} \chi,
$$

where $\chi$ denotes the combined shadowing and fading component of the interference statistics.

4) Derive the MGF of the cumulative interference $Y=$ $\sum_{l=1}^{L} X_{l}$ caused by the allocated interfering users.

In this work, we assume a proportional fair scheduling scheme which allocate the subcarrier to the user with largest normalized SNR $(\gamma / \bar{\gamma})[10]$, where $\gamma$ and $\bar{\gamma}$ denote the instantaneous SNR and the short term average SNR of a given user, respectively. In other words, the selection criteria is based on selecting a user who has maximum instantaneous SNR relative to its own average SNR. It is important to note that the performance of proportional fair scheduling schemes is independent of the path loss factor if users are moving relatively slowly, i.e., their path loss remains nearly the same on a short term basis. In this case, using (1), $\zeta / \bar{\zeta}$ becomes equal to $\gamma / \bar{\gamma}$ where $\bar{\zeta}=\int_{0}^{\infty} \zeta f_{\zeta}(\zeta) d \zeta$ is the average of the composite fading statistics. Moreover, the users that are co-located are more likely to possess similar fading severity. Therefore, we assume independent and identically distributed (i.i.d.) channel statistics for users located within a given circular region. The users located in different circular regions are assumed to have non-identically distributed channel gains.

\section{Distribution of the Allocated User Locations}

We present an approach to derive the discrete distribution of the distance of allocated user in a given cell, i.e., the probability mass function (PMF) of $r_{\text {sel }}$ based on proportional fair scheduling. The derivation is divided into two steps.

Step 1 (Selecting the user with maximum normalized SNR in ring $k$ ): As each user within a ring $k$ is assumed to have constant short term path loss, the problem can be stated as:

$$
\begin{aligned}
\frac{\gamma_{k}}{\bar{\gamma}_{k}} & =\max \left\{\frac{\gamma_{1}}{\bar{\gamma}_{1}}, \frac{\gamma_{2}}{\bar{\gamma}_{2}}, \cdots, \frac{\gamma_{i}}{\bar{\gamma}_{i}}, \cdots, \frac{\gamma_{u_{k}}}{\bar{\gamma}_{u_{k}}}\right\} \\
& =\max \left\{\frac{\zeta_{1}}{\bar{\zeta}_{1}}, \frac{\zeta_{2}}{\bar{\zeta}_{2}}, \cdots, \frac{\zeta_{i}}{\bar{\zeta}_{i}}, \cdots, \frac{\zeta_{u_{k}}}{\bar{\zeta}_{u_{k}}}\right\} .
\end{aligned}
$$

As the channel gain of the users located within a circular region are assumed to be i.i.d., the problem of selecting the user with maximum normalized SNR becomes equal to selecting the user with maximum instantaneous SNR, i.e.,

$$
\gamma_{k}=\max \left\{\zeta_{1}, \zeta_{2}, \cdots, \zeta_{i}, \cdots, \zeta_{u_{k}}\right\}
$$

where, $\gamma_{k}$ is the SNR of the selected user in ring $k$. Thus, for any ring $k$, the cumulative distribution function (CDF) and PDF of $\gamma_{k}$ can be written as follows:

$$
\begin{gathered}
F_{\gamma_{k}}\left(\gamma_{k}\right)=\prod_{i=1}^{u_{k}} F_{\zeta_{i}}\left(\gamma_{k}\right)=\left(F_{\zeta}\left(\gamma_{k}\right)\right)^{u_{k}} \\
f_{\gamma_{k}}\left(\gamma_{k}\right)=u_{k} f_{\zeta}\left(\gamma_{k}\right)\left(F_{\zeta}\left(\gamma_{k}\right)\right)^{u_{k}-1}
\end{gathered}
$$

However, it is important to consider that the selected users located in different circular regions possess non i.i.d. channel gains and hence possess different $\overline{\gamma_{k}}$. This factor can be incorporated simply by letting $\xi_{k}=\frac{\gamma_{k}}{\bar{\gamma}_{k}}$ and performing the transformation of RVs. Thus, the CDF and PDF of $\xi_{k}$ can be written as follows:

$$
\begin{gathered}
F_{\xi_{k}}\left(\xi_{k}\right)=\left(F_{\gamma_{k}}\left(\bar{\gamma}_{k} \xi_{k}\right)\right)^{u_{k}} . \\
f_{\xi_{k}}\left(\xi_{k}\right)=\frac{u_{k}}{\bar{\gamma}_{k}} f_{\gamma_{k}}\left(\bar{\gamma}_{k} \xi_{k}\right)\left(F_{\gamma_{k}}\left(\xi_{k} \bar{\gamma}_{k}\right)\right)^{u_{k}-1} .
\end{gathered}
$$

The short term average SNR $\bar{\gamma}_{k}$ of the selected user in each ring $k$ can be evaluated as follows:

$$
\bar{\gamma}_{k}=\int_{0}^{\infty} \gamma_{k} f_{\gamma_{k}}\left(\gamma_{k}\right) d \gamma_{k} .
$$

Step 2 (Selecting the user with maximum normalized SNR among $K$ rings): In this step, we compute the probability of selecting the $k^{\text {th }}$ ring among all rings. This is equivalent to selecting the ring $k$ which possesses the user with the highest normalized SNR $\xi_{k}$ among all rings. Thus, conditioning on $\xi_{k}$, the PDF of $r_{\text {sel }}$ can be written as follows:

$$
P\left(r_{\mathrm{sel}}=r_{k} \mid \xi_{k}\right)=\prod_{i=1, i \neq k}^{K} p\left(\xi_{i} \leq \xi_{k}\right)=\prod_{i=1, i \neq k}^{K} F_{\xi_{i}}\left(\xi_{k}\right) \text {. }
$$

By averaging over the distribution of $\xi_{k}$, the PMF of $r_{\text {sel }}$ can be written as follows:

$$
P\left(r_{\mathrm{sel}}=r_{k}\right)=\int_{0}^{\infty}\left(\prod_{i=1, i \neq k}^{K} F_{\xi_{i}}\left(\xi_{k}\right)\right) f_{\xi_{k}}\left(\xi_{k}\right) d \xi_{k},
$$

where $r_{\text {sel }} \in[0, R]$. The result in (13) is independent of any particular shadowing and fading statistics. Although, (13) is not a closed form expression, the integration can be solved accurately using standard software packages such as MAPLE and MATHEMATICA.

Note that $P\left(r_{\text {sel }}=r_{k}\right)$ in (13) is the marginal PMF of $P\left(r_{\text {sel }}=r_{k}, \theta=\theta_{n}\right)$ where $\theta$ is distributed uniformly from 0 to $2 \pi$. Although the RV $\theta$ possesses continuous distribution, we discretize it in order to reduce complexity. Thus, discretizing the range of RV $\theta$ into $N$ uniform angular intervals, $P\left(\theta=\theta_{n}\right)$ is $1 / N$, where $\theta_{n}$ denotes any discrete value that the RV $\theta$ can take. Since $r_{\text {sel }}$ and $\theta$ are independent, their joint PMF can be written as follows:

$$
P\left(r_{\text {sel }}=r_{k}, \theta=\theta_{n}\right)=\frac{P\left(r_{\text {sel }}=r_{k}\right)}{N},
$$

\section{Distribution OF THE ICI}

The derivation for the distribution of ICI from an interfering cell $l$, i.e., $f_{X_{l}}(x)$ depends on the distribution of the distance of the allocated users in the interfering cell $l$ to the BS of interest, i.e., $f_{\tilde{r}_{\text {sel }_{l}}}(\tilde{r})$. As mentioned earlier, each interfering 


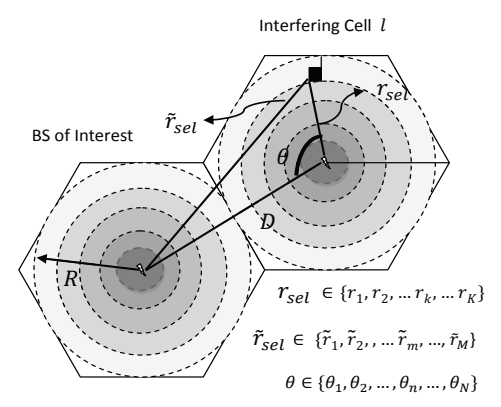

Fig. 1. Cellular network model with each cell divided into multiple rings.

cell is assumed to have identical conditions and, thus, $f_{\tilde{r}_{\mathrm{sel}}}(\tilde{r})$ remains the same for all interfering cells. Therefore, we will drop the subscript $l$ in the sequel to simplify notation.

Using the cosine law, we can write:

$$
\tilde{r}_{\mathrm{sel}}^{2}=r_{\mathrm{sel}}^{2}+D^{2}-2 r_{\mathrm{sel}} D \cos \theta,
$$

where $\tilde{r}_{\text {sel }}$ is the distance of the allocated user in the interfering cell $l$ from the BS of interest, $r_{\text {sel }}$ is the distance of the allocated user from its own BS, i.e., (BS $l$ ), $\theta \in[0,2 \pi]$ and $D=2 R$ since we consider universal frequency reuse with one tier of interfering cells. The approach can be extended to any number of tiers in a straightforward manner.

To derive the PMF of $\tilde{r}_{\text {sel }}$ where $\tilde{r}_{\text {sel }} \in[D-R, D+R]$, firstly we compute $\tilde{r}_{n, k}$ for given $\theta_{n}$ and $r_{k}$ using (15) as follows:

$$
\tilde{r}_{n, k}^{2}=r_{k}^{2}+D^{2}-2 r_{k} D \cos \theta_{n} \quad \forall r_{k}, \forall \theta_{n} .
$$

Clearly $\tilde{r}_{n, k}$ are the points at which $P\left(\tilde{r}_{\text {sel }}=\tilde{r}_{n, k}\right)$ can be defined using (14) as follows:

$$
P\left(\tilde{r}_{\mathrm{sel}}=\tilde{r}_{n, k}\right)=\frac{P\left(r_{\mathrm{sel}}=r_{k}\right)}{N} .
$$

The two dimensional data set of $\tilde{r}_{\text {sel }}$, at which $P\left(\tilde{r}_{\text {sel }}=\tilde{r}_{n, k}\right)$ is defined, can then be grouped into $M$ segments of any arbitrary width $\Delta$. This can be done by dividing the distance between $D-R$ and $D+R$ into $M$ equal segments of width $\Delta$ and mapping $\tilde{r}_{n, k}$ accordingly. Clearly, by adding all the probabilities for which $\tilde{r}_{\text {sel }}$ lies in the $m^{\text {th }}$ segment we get the probability of $\tilde{r}_{\text {sel }}=\tilde{r}_{m}$ as follows:

$$
P\left(\tilde{r}_{\text {sel }}=\tilde{r}_{m}\right)=\sum_{\tilde{r}_{n, k} \in\left[\tilde{r}_{m}-\frac{\Delta}{2}, \tilde{r}_{m}+\frac{\Delta}{2}\right]} P\left(\tilde{r}_{\text {sel }}=\tilde{r}_{n, k}\right),
$$

where $\tilde{r}_{m}$ denotes any discrete value that the RV $\tilde{r}_{\text {sel }}$ can take (see Fig. 1). Now, using (3) the PDF of $X$ conditioned on $\tilde{r}_{\text {sel }}$ can be determined by RV transformation as follows:

$$
f_{X \mid \tilde{r}_{\mathrm{sel}}}=\frac{f_{\chi}\left(x \tilde{r}_{\mathrm{sel}}^{\beta}\right)}{\tilde{r}_{\mathrm{sel}}^{-\beta}} .
$$

Averaging (19) over the PMF of $\tilde{r}_{\text {sel }}$ the distribution of interference, i.e., $f_{X}(x)$ from any interfering cell $l$ can be written as follows:

$$
f_{X}(x)=\sum_{\tilde{r}_{m}=\tilde{r}_{1}}^{\tilde{r}_{M}} \frac{f_{\chi}\left(x \tilde{r}_{m}^{\beta}\right)}{\tilde{r}_{m}^{-\beta}} P\left(\tilde{r}_{\text {sel }}=\tilde{r}_{m}\right) .
$$

Deriving the distribution of $Y$ requires the convolution of RVs $X_{l}$ for $l=1,2, \cdots L$. However, assuming that all interferers are i.i.d., the MGF $\mathcal{M}_{Y}(t)$ of $Y$ can be written simply as:

$$
\mathcal{M}_{Y}(t)=\prod_{l=1}^{L} \mathcal{M}_{X_{l}}(t)=\left(\mathcal{M}_{X}(t)\right)^{L}=\left(\mathbb{E}\left[e^{t x}\right]\right)^{L} \text {. }
$$

Looking at the structure of (20), we can write $\mathcal{M}_{X}(t)$ as follows:

$$
\mathcal{M}_{X}(t)=\sum_{\tilde{r}_{m}=\tilde{r}_{1}}^{\tilde{r}_{M}} \frac{P\left(\tilde{r}_{\mathrm{sel}}=\tilde{r}_{m}\right)}{\tilde{r}_{m}^{-\beta}} \int_{0}^{\infty} e^{t x} f_{\chi}\left(x \tilde{r}_{m}^{\beta}\right) d x .
$$

The expression in (22) is general and applies to various channel models. The main motivation underlying the development of MGF based framework is to reduce the intricacy in the analytical evaluation of various system performance metrics. Next, we study the interference statistics for two typical fading scenarios.

Example 1 (Rayleigh Fading - $\zeta, \chi \sim \operatorname{Exp}(\lambda)$ ): Considering the simplest case of Rayleigh fading with no shadowing $f_{X}(x)$ can be written using (20) as follows:

$$
f_{X}(x)=\sum_{\tilde{r}_{m}=\tilde{r}_{1}}^{\tilde{r}_{M}} \lambda \tilde{r}_{m}^{\beta} e^{-\lambda \tilde{r}_{m}^{\beta} x} P\left(\tilde{r}_{\text {sel }}=\tilde{r}_{m}\right) .
$$

Note that (23) is a hyper-exponential distribution with parameter $\lambda \tilde{r}_{m}^{\beta}$. Thus, using MGF of the hyper-exponential distribution, $\mathcal{M}_{Y}(t)$ can be derived as follows:

$$
\mathcal{M}_{Y}(t)=\left(\sum_{\tilde{r}_{m}=\tilde{r}_{1}}^{\tilde{r}_{M}} \frac{\lambda \tilde{r}_{m}^{\beta}}{\lambda \tilde{r}_{m}^{\beta}-t} P\left(\tilde{r}_{\text {sel }}^{\beta}=\tilde{r}_{m}\right)\right)^{L} .
$$

Example 2 (Composite Fading - $\zeta, \chi \sim \operatorname{Gamma}\left(m_{s}, m_{c}\right)$ ): In this example, another important scenario is considered in which shadowing and fading statistics are modeled by a Gamma and Nakagami distribution, respectively. In this case, the composite distribution has a closed form which is known as generalized-K or Gamma-Gamma distribution [11]. In order to avoid the computational difficulties, while dealing with the generalized-K RV, the authors in [12] proposed an accurate approximation of the generalized-K RV using moment matching method, i.e., the generalized-K distribution can be approximated by a simple Gamma distribution using moment matching method [12]. Therefore in this case, using (20), $f_{X}(x)$ can be written as follows:

$$
f_{X}(x)=\sum_{\tilde{r}_{m}=\tilde{r}_{1}}^{\tilde{r}_{M}} \frac{e^{-\frac{x \tilde{r}_{m}^{\beta}}{m_{c}}}\left(x \tilde{r}_{m}^{\beta}\right)^{m_{s}-1}}{\tilde{r}_{m}^{-\beta} \Gamma\left(m_{s}\right) m_{c}^{m_{s}}} P\left(\tilde{r}_{s e l}=\tilde{r}_{m}\right) .
$$

Therefore by some manipulations, $\mathcal{M}_{Y}(t)$ can be derived as follows:

$$
\mathcal{M}_{Y}(t)=\left(\sum_{\tilde{r}_{m}=\tilde{r}_{1}}^{\tilde{r}_{M}} P\left(\tilde{r}_{\mathrm{sel}}=\tilde{r}_{m}\right)\left(\frac{\tilde{r}_{m}^{\beta}}{\tilde{r}_{m}^{\beta}-m_{c} t}\right)^{m_{s}}\right)^{L} .
$$

\section{Network Performance Metrics}

In this section, we explain how the derived expressions can be used to compute the outage probability $P_{\text {out }}$ and average fairness measure $\mathcal{F}$ in the network. The computation of the network ergodic capacity $\mathcal{C}$ is skipped due to space limitations; however, it is detailed in [13].

\section{A. Evaluation of Outage Probability}

To compute $P_{\text {out }}$, we follow the strategy introduced in [14]. Firstly, a new RV $Z=q \sum_{l=1}^{L} X_{l}-X_{0}$ is defined, where $q$ is the outage threshold and $\bar{X}_{0}$ is the corresponding signal power of the user in the cell of interest. The outage event is defined as $p(Z \geq 0)$. The characteristic function of $Z$ can be written as $\phi_{Z}(\omega)=\mathbb{E}\left[e^{j Z \omega}\right]=\phi_{Y}(q \omega) \phi_{X_{0}}(-\omega)$. Finally, $P_{\text {out }}$ can be computed by evaluating the definite integral [14] as follows:

$$
P_{\text {out }}=\frac{1}{2}+\frac{1}{2 \pi} \int_{-\infty}^{\infty} \operatorname{Im}\left(\frac{\phi_{Z}(\omega)}{\omega}\right) d \omega,
$$


where $\operatorname{Im}\left(\phi_{Z}(\omega)\right)$ denotes the imaginary part of $\phi_{Z}(\omega)$. The expression for $\phi_{Z}(\omega)$ is presented for the second example scenario with composite fading as follows:

$$
\begin{aligned}
\phi_{Z}(\omega)= & \left(\sum_{\tilde{r}_{m}=\tilde{r}_{1}}^{\tilde{r}_{M}}\left(\frac{\tilde{r}_{m}^{\beta}}{\tilde{r}_{m}^{\beta}-j q \omega m_{c}}\right)^{m_{s}} P\left(\tilde{r}_{\text {sel }}=\tilde{r}_{m}\right)\right)^{L} \\
& \times \sum_{r_{k}=r_{1}}^{r_{K}}\left(\frac{r_{k}^{\beta}}{r_{k}^{\beta}+j \omega m_{c_{k}}}\right)^{m_{s_{k}}} P\left(r_{\text {sel }}=r_{k}\right),
\end{aligned}
$$

where, $m_{s_{k}}$ and $m_{c_{k}}$ are the shape and scale parameters of $\zeta$. Since the statistics of $\zeta$ is assumed to be non-identically distributed among the rings, therefore the parameters varies from ring to ring. Using (28), it is straightforward to write the expression for the first example scenario with Rayleigh fading.

\section{B. Evaluation of Average Fairness}

In order to quantify the degree of fairness among different scheduling schemes, we use the notion developed in [15]. The average fairness of a resource allocation scheme in a network with $U$ users can be written as follows:

$$
\mathcal{F}=-\sum_{i=1}^{U} p_{i} \frac{\log p_{i}}{\log U}
$$

where $p_{i}$ is the proportion of resources allocated to a user $i$ or the access probability of user $i$. A system is strictly fair if each user has equal probability to access the channel and in such case the average fairness becomes one. The other extreme occurs when the channel access is dominated by a single user; in such case, the average fairness reduces to zero. The average fairness can be easily computed using our derived results as :

$$
\mathcal{F}=-\sum_{k=1}^{K} P\left(r_{\text {sel }}=r_{k}\right) \frac{\log \cdot P\left(r_{\text {sel }}=r_{k}\right)-\log u_{k}}{\log U}
$$

\section{RESUlts AND ANALYSiS}

In this section, we will substantiate the accuracy of the derived expressions through Monte Carlo simulations. Also, we analyze and compare the performance of the proportional fair scheduler with the greedy scheduler. Due to the lack of space, the results are presented for the second example scenario with composite fading; however, the trend remains similar for the Rayleigh fading scenario. The radius $R$ of the cell is taken $500 \mathrm{~m}$ and the path loss exponent $\beta=3$. The cell is decomposed into non-uniform circular regions of width $\Delta_{k}$ each. The path loss variation within each region is set equal to $2 \mathrm{~dB}$. It is important to note that the composite fading statistics $\operatorname{Gamma}\left(m_{s}, m_{c}\right)$ of the signal $X_{0}$ is assumed to vary from ring to ring. More precisely $m_{s}=1$ in the very first ring and continues to decrease from cell center to cell edge linearly with a step of 0.01 . Thus, the users located in different rings have non-identically distributed composite fading statistics. The interfering statistics $f_{\chi}(\chi)$ is set as $\operatorname{Gamma}(3 / 2,2 / 3)$.

Firstly, we explain the Monte Carlo simulation setup used for evaluating the derived distributions.

1) Generate $U$ uniformly distributed users per cell. Each user has an instantaneous SNR given by (1).

2) Greedy scheduling: Select a user from each ring with maximum instantaneous SNR.

3) Proportional fair scheduling: Select a user from each ring with maximum instantaneous shadowing and fading irrespective of the path loss.

4) Select two users among all rings, one with maximum SNR (for greedy scheme) and one with maximum normalized SNR (for proportional fair scheme). Note that the short term average SNR for each selected user in a ring is given by (11).
5) Store the distance of the two selected users, i.e., $r_{\text {sel }}$ from their serving BS.

6) Compute the distance of these two selected users from the BS of interest, i.e., $\tilde{r}_{\text {sel }}$ using cosine law and finally generate the interference using (3).

7) Repeat all steps for a large number of iterations (in this section, we run 100,000 iterations). Generate histogram for the discrete RV $r_{\text {sel }}$ with non-uniform bin widths and another histogram for the discrete RV $\tilde{r}_{\text {sel }}$ with any arbitrary bin width equal to $\Delta$ (we take $\Delta=50 \mathrm{~m}$ ).

Fig. 2 depicts the PMF of the distance of the allocated user in any given cell assuming proportional fair scheduling with maximum normalized SNR selection. The results are presented for 50 and 200 users. In order to get an integer number of users in each region, we perform rounding, i.e., we consider zero users in the regions where the average number of users are less than half. Thus, the probability of allocating a user in these regions is zero both in the analytic evaluation and in the simulations. Since the proportional fair scheduling scheme inherits some fairness among users in a cell, the PMF of the allocated user locations is expected to be more flat compared to greedy scheduling as demonstrated in Fig. 3 .

It can be observed that for $U=50$, the PMF starts from $0.09 \mathrm{~km}$ whereas for $U=200$ the corresponding PMF starts from $0.05 \mathrm{~km}$. Clearly, low number of users leads to ignoring more rings near the cell center. It can also be observed that as the number of users increases, the greater is the chance of allocating a user near the cell center. The obtained PMF results fit well with exhaustive Monte Carlo simulation results which verifies the accuracy of the derived expressions.

In Fig. 4, the PMF of the distance at which users are allocated in the interfering cell $l$, i.e., $P\left(\tilde{r}_{\text {sel }}=\tilde{r}_{m}\right)$ is shown. Simulation results are found to be in agreement with the analytical results. As it is more likely that a user near its serving BS can get a subcarrier, thus the PMF of the allocated interfering users is expected to have high density in the middle. However, the slight descend in the middle is due to ignoring any user within the rings where the average number of users becomes less than half.

Fig. 5 demonstrates the outage probability in the network for 50 users. The performance of both the greedy and the proportional fair scheduling schemes are compared for various path loss exponents. As expected, the greedy scheduler achieves less outage, but higher throughput gains [13]. Moreover, as $\beta$ increases, the outage probability in the network decreases due to rapid signal degradation which leads to interference reduction. Fig. 6 quantifies the average fairness for the greedy and proportional fair scheduling schemes. Clearly, the proportional fair scheduling scheme provides higher fairness than the greedy scheduling scheme.

\section{CONCLUSION}

This paper presents a semi-analytical approach to model the uplink ICI considering proportional fair scheduling. The presented approach does not require the knowledge of instantaneous scheduling decisions and applies to a wide range of channel models. The accuracy of the derived expressions is verified via Monte Carlo simulations. The derived expressions are shown to be also useful to evaluate important network performance metrics that include outage probability and network fairness.

\section{REFERENCES}

[1] B. Pijcke, M. Z-Colin, M. Gazalet, M. Gharbi, and P. Corlay, "An analytical model for the intercell interference power in the downlink of wireless cellular networks," arXiv:1107.4199v1, July, 2011.

[2] K.W. Sung, H. Haas, and S. McLaughlin, "A semianalytical PDF of downlink SINR for femtocell networks," EURASIP Journal on Wireless Communications and Networking, vol. January, 2010.

[3] S.H. Kim, B.C. Jung, and D.K. Sung, "Effect of other-cell interference on multiuser diversity in cellular networks," International Symposium on Communications and Information Technologies. ISCIT'07, pp. 521-525, Sydney, Australia, October, 2007. 
[4] S. Plass, X.G. Doukopoulos, and R. Legouable, "Investigations on linklevel inter-cell interference in OFDMA systems," IEEE Symposium on Communications and Vehicular Technology, pp. 49-52, Liege, November, 2006.

[5] S. Elayoubi, B. Haddada, and B. Fourestie, "Performance evaluation of frequency planning schemes in OFDMA based networks," IEEE Transactions on Wireless Communications, vol. 7, no. 5, pp. 1623-1633, May, 2008.

[6] R. Kwan and C. Leung, "On collision probabilities in frequency-domain scheduling for LTE cellular networks," IEEE Communication Letters, vol. 15, no. 9, pp. 965-967, September, 2011.

[7] E.Oh, M.G. Cho, S. Han, C. Woo, and D. Hong, "Performance analysis of reuse-partitioning-based subchannelized OFDMA uplink systems in multicell environments," IEEE Transactions on Vehicular Technology, vol. 57, no. 4, pp. 2617-2621, July, 2008.

[8] I.Viering, A. Klein, M. Ivrlac, M. Castaneda, and J.A. Nossek, "On uplink intercell interference in a cellular system," IEEE International Conference on Communications, ICC'06, vol. 5, pp. 2095-2100, Istanbul, Turkey, June, 2006

[9] H. Tabassum, F. Yilmaz, Z. Dawy, and M-S. Alouini , "On the statistics of uplink inter-cell interference with greedy resource allocation," to appear in IEEE International Symposium on Wireless Communication Systems, ISWCS, Aachen, Germany, November, 2011.

[10] N.Sharma and H.L.Ozarow, "A study of opportunism for multipleantenna systems," IEEE Transactions on Information Theory, vol. 51, no. 5, pp. 1804-1814, May, 2005.

[11] I. M. Kostic, "Analytical approach to performance analysis for channel subject to shadowing and fading," IEE Proceedings Commmunication, vol. 152 , no. 6 , pp. 821-827, 2005.

[12] S. Al-Ahmadi and H. Yanikomeroglu, "On the approximation of the generalized-K PDF by a Gamma PDF using the moment matching method," Wireless Communications and Networking Conference, WCNC'09, pp. 1-6, Budapest, Hungary, April 2009.

[13] H. Tabassum, F. Yilmaz, Z. Dawy, and M-S. Alouini , "Statistical Modeling of Uplink Inter-Cell Interference based on scheduling," Uploaded to arXiv in Advance of Publication, 2011.

[14] Q.T. Zhang, "Outage probability of cellular mobile radio in the presence of multiple Nakagami interferers with arbitrary fading parameters" IEEE Transactions on Vehicular Technology, vol. 44, no. 3, pp. 364-372, May, Transacti

[15] R. Elliott, "A measure of fairness of service for scheduling algorithms in multiuser systems," Proceedings of IEEE Canadian Conference on Electrical and Computer Engineering, CCECE, pp. 1583-1588, Winnipeg, Canada, May, 2002.
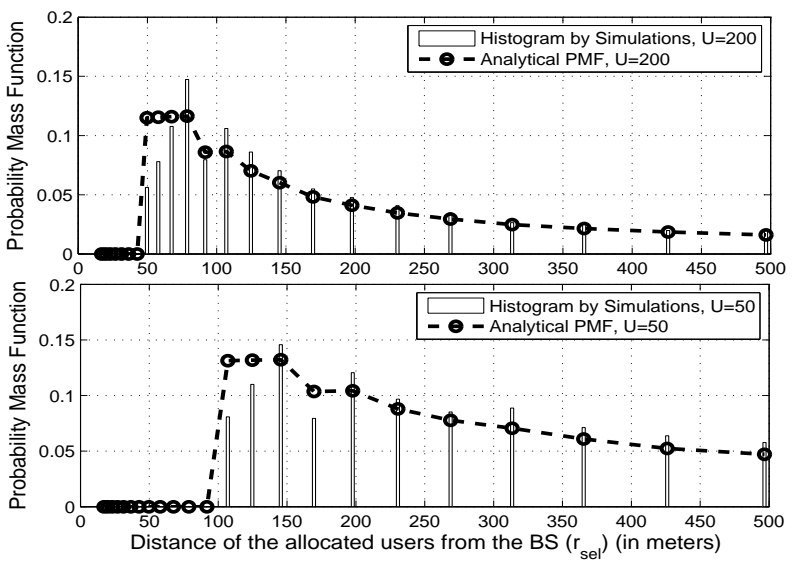

Fig. 2. PMF of the distance of allocated users in a cell (i.e., PMF of $r_{\text {sel }}$ ).
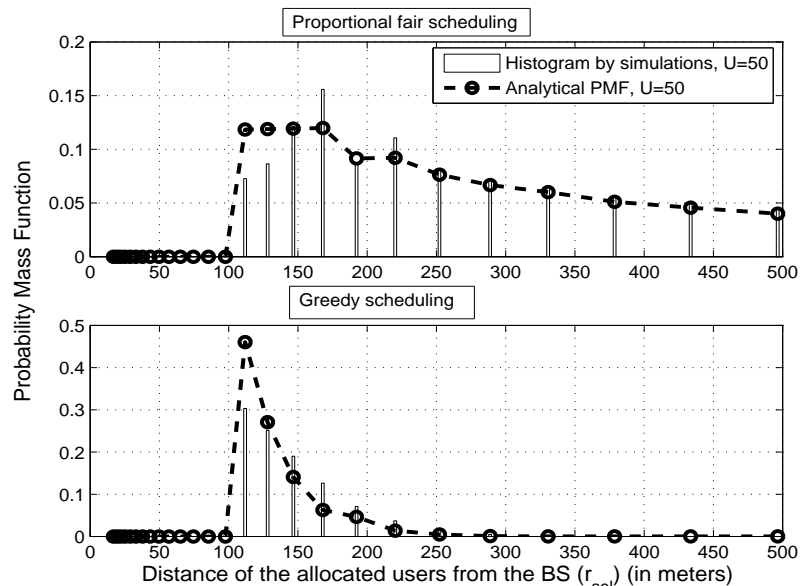

Fig. 3. A comparison of the PMF of the distance of the allocated user for greedy and proportional fair scheduler, $\mathrm{U}=50$.

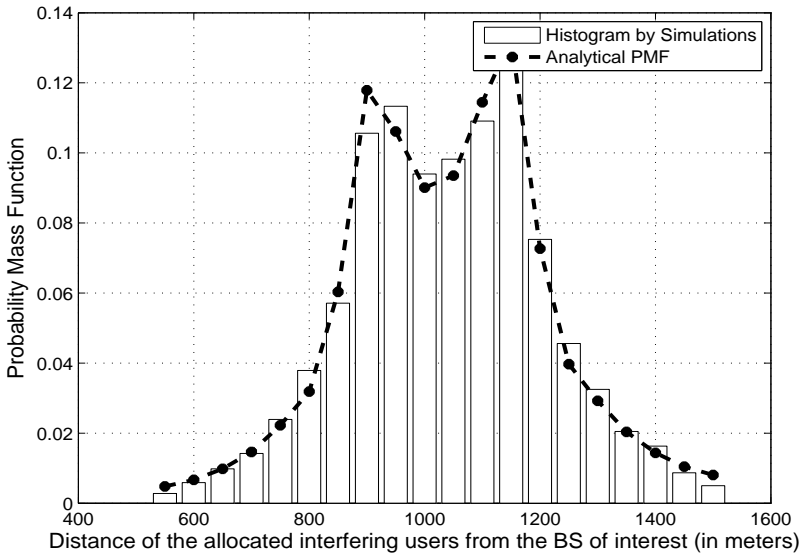

Fig. 4. PMF of the distance at which interfering users are allocated (i.e., PMF of $\left.\tilde{r}_{\mathrm{sel}}\right), N=720$

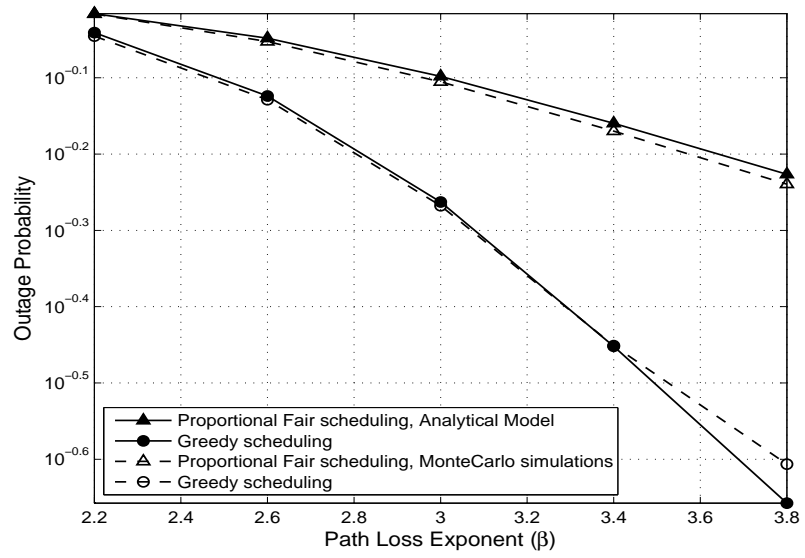

Fig. 5. Outage probability of the network $\left(P_{\text {out }}\right)$ against path loss exponent $(\beta)$ for greedy and proportional fair scheduler, $q=18 \mathrm{~dB}$.

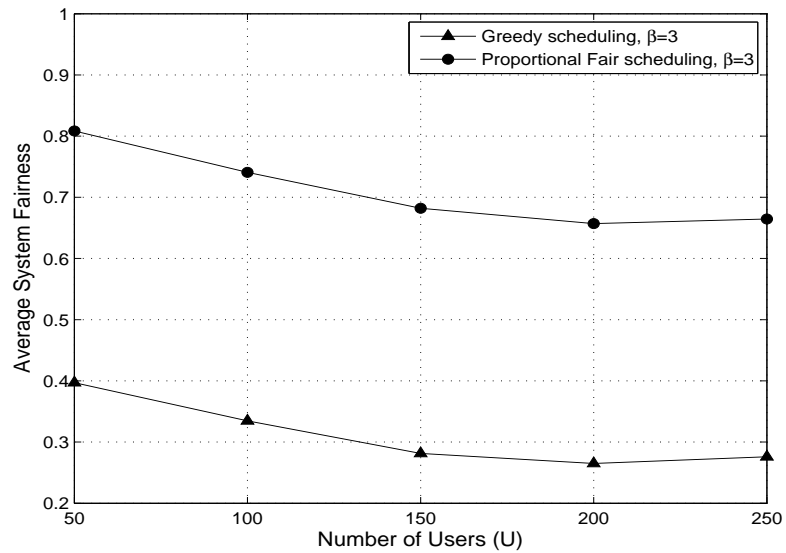

Fig. 6. Average system fairness of greedy and proportional fair scheduling schemes for different number of users. 